\title{
Wolf-Rayet populations in starburst galaxies
}

\author{
Richard P.F. Norris, Linda J. Smith, and Paul A. Crowther \\ Department of Physics and Astronomy, University College London, \\ Gower Street, London WC1E 6BT, UK
}

\begin{abstract}
Recent advances in atmosphere codes now permit the calculation of realistic grids of hot star atmospheres. Here we use the new $O$ and WR star atmosphere of Smith, Norris \& Crowther (2002) with an updated version of the evolutionary synthesis code STARBURST 99 to predict the population of WR stars in a variety in astrophysical conditions. We present a series of synthetic starburst spectra in the optical region which shows the time evolution of the WR-bumps at $\sim 4650 \AA$ and $\sim 5800 \AA$.
\end{abstract}

Wolf-Rayet populations are important indicators of the age, metallicity and Initial Mass Function (IMF) of a particular starbursting system. Creating a synthetic spectrum of WR-bumps at $4686 \AA$ and $5808 \AA$ could directly probe the underlying WR population of a starburst. We have updated the population synthesis code STARBURST99 of Leitherer et al. (1999), by including a new set of O-type star atmospheres and replacing the unblanketed WR models of Schmutz et al. (1992). These new grids were calculated using the non-LTE line-blanketed atmosphere codes of Pauldrach et al. (2001, WM-BASIC) and Hillier \& Miller (1998, CMFGEN), respectively, as described by L.J. Smith (these Proceedings) and Smith et al. (2002). Here we concentrate on the applications of the WR grid, which spans 5 metallicities from 0.05 to $2 \mathrm{Z}_{\odot}$, with the inclusion of WN and WC stars modelled over a range of effective temperatures from 40 to $140 \mathrm{kK}$. These new atmosphere grids represent a significant improvement over the pure helium WR models of Schmutz et al. (1992).

Using the original high-resolution CMFGEN grid to represent both the WN and WC subspecies in the STARBURST99 code, we have generated synthetic spectra to represent the Wolf-Rayet phase of a starburst. We include the WR and O-type supergiant contributions, since the latter have a significant effect on the blue WR-bump at early ages $(<3.0 \mathrm{Myr})$ due to their strong winds.

It is also possible to create time series, to model the evolution of the WR bump profiles over time. Figure 1 shows the WR contribution to the spectra for the first $4.5 \mathrm{Myr}$ of an instantaneous, solar metallicity burst with an IMF with $M_{\mathrm{up}}=100 \mathrm{M}_{\odot}$ and a Salpeter $\alpha$ exponent. This figure shows the narrow emission line contribution from the dominant O-type supergiant population creating strong N IV $\lambda 4057$ and N III $\lambda 4640$ features in the starburst continuum. These supergiant models were calculated using CMFGEN to take advantage of its comoving frame geometry, which can accurately model emission-line formation in dense stellar winds. At later times, the narrow absorption and emission line contribution disappears with the O-type supergiants, and the broader features 


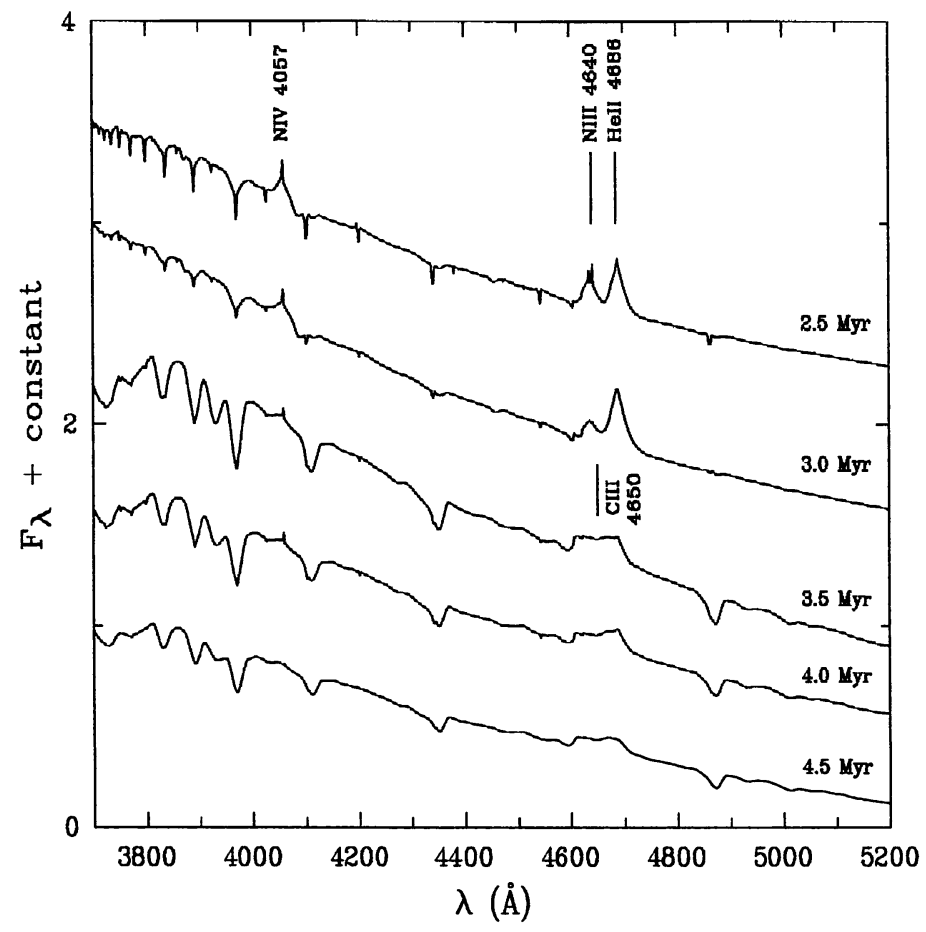

Figure 1. The time evolution of the blue WR-bump from 2.5 to $4.5 \mathrm{Myr}$ for a solar metallicity instantaneous burst.

of the WR population appear. The blue WR-bump shown at ages $<3.5 \mathrm{Myr}$ is a blend of He II $\lambda 4686$ and $\mathrm{N}$ III $\lambda 4640$, arising mainly from the late type WN population. At later ages, the WC population dominates the starburst and the blue WR bump is mainly due to $\operatorname{CIII} \lambda 4650$.

In the future,we intend to compare our synthetic spectra with WR galaxy observations to derive ages, WR/O and WC/WN number ratios, and metallicities. This technique should be more accurate than the current WR-bump equivalent width method (e.g., Schaerer \& Vacca 1998).

\section{References}

Hillier, D.J., Miller, D.L. 1998, ApJ 496, 407

Leitherer, C., Schaerer, D., Goldader, J.D. 1999, ApJS 123, 3

Pauldrach, A.W.A., Hoffmann, T.L., Lennon, M. 2001, A\&A 375, 161

Schaerer, D., Vacca, W.D., 1998, ApJ 497, 618

Schmutz, W., Leitherer, C., Gruenwald, R. 1992, PASP 102, 1164

Smith, L.J., Norris, R.P.F., Crowther, P.A. 2002, MNRAS 337, 1309 